\title{
XLIV. Experimental error in calorimetric work
}

\section{Spencer Umfreville Pickering M.A.}

To cite this article: Spencer Umfreville Pickering M.A. (1886) XLIV. Experimental error in calorimetric work, Philosophical Magazine Series 5, 21:131, 324-330, DOI: 10.1080/14786448608627853

To link to this article: http://dx.doi.org/10.1080/14786448608627853

曲 Published online: 08 Jun 2010.

Submit your article to this journal

Џ Article views: 2

Q View related articles $\sqsubset$ 
variations of $\tan \frac{\theta}{\alpha}$ is

extending from $+\infty$ to $-\infty$.

$$
y=\frac{1}{\pi} \frac{1}{\left(1+y^{2}\right)},
$$

In such cases the general proof of the law of error breaks down. The general formula for the modulus, that it is the double sum of squares of the apparent errors, is apt to yield an infinite result. We must fall back upon the explicit methods. If, for instance, the law of facility above written, or rather the more general form

$$
y=\frac{1}{\pi} \frac{s}{s^{2}+y^{2}}
$$

held good for any species of phenomena, then, in order to determine the improbability of a chance deviation to the extent of $y^{\prime}$, we must integrate $y$ from $\infty$ to $y^{\prime}$.

In view of the rarity of such cases, I think we are justified in concluding that the received formula enounced at the outset is in general either accurate or at least safe; and that, in the exceptional case where it is neither correct nor safe, it is still corrigible, so far as the laws of error which exist in nature may be regarded as binomials.

XLIV. Erperimental Error in Calorimetric Work. By Spencer Umfreville Pickering, M.A., Professor of Chemistry at Bedford College*.

THE determination of the heat of dissolution of a solid in water is an operation which will always occupy a most important position in the majority of calorimetric determinations; and it is therefore desirable to ascertain what the experimental error involved in this process is, and in what direction we must look for a reduction of this error. The large number of these determinations which I have had occasion to make during the last two years will give the means requisite for an investigation of this error.

The modus operandi in the experiments was as follows:The given salt was weighed out into test-tubes on feet, which wero placed in an open metal beaker carefully guarded from radiation and draughts by screens; in this was suspended a delicate thermometer, a companion instrument to that used in the calorimeter itself, the exact difference in the readings

* Communicated by the Physical Society : read February 13, 1886. 
between the two instruments being known throughout the extent of their scales.

It was found that one hour * was a sufficient interval to allow for the salt to assume the temperature of the surrounding atmosphere, provided this latter remained constant during this time. In order to diminish the error in measuring the rate of cooling (or heating) during the dissolution of the salt, the temperature of the air was always maintained as nearly as possible at the temperature of the calorimetric water, the difference in the majority of cases not exceeding $\cdot 1^{\circ} \mathrm{C}$, or at the most $\cdot 2^{\circ} \mathrm{C}$. The correction to be applied, owing to the temperature of the salt not being exactly the same as that of the calorimetric water, was thus rendered very small, and in most cases quite negligible.

The alteration in the temperature of the salt during its removal to the calorimeter ean scarcely be appreciable, since this operation does not take more than ten or fifteen seconds.

The calorimeter employed was an open calorimeter similar to that devised by Berthelot (Mécanique Chimique, vol. i. p. 140); a pounder such as Berthelot uses to crush salts which cake (ibid. vol. i. p. 183) being employed in all cases, not only as a pounder, but also as a stirrer, and the platinum vessel in which the operations were performed being always used without its cover ; indeed, free circulation of air round the calorimeter is most important in all delicate determinations. It was found that an annular cardboard disk, covering in a most imperfect manner the space between the calorimeter and the surrounding water-jacket, enclosed the air to an extent sufficient to make it act as so much calorimetric water, and introduced a very appreciable error into the results.

In dissolving an anhydrous salt, when eaking generally occurs, the calorimeter proper cannot be placed on the cork tripod as in other cases, but must be supported on a cork disk covered with cardboard; and, inasmuch as this disk was found to absorb an appreciable amount of heat from the calorimeter, the quantity thus absorbed had to be determined by performing a series of experiments in which a reaction of known calorimetric value was effected in the calorimeter when supported on the tripod and on the disk alternately. The we of this disk unfortunately introduces a small experimental error, since from continual pounding the form of the bottom of the

* A hygroscopic substance may be left in a tube well closed with an india-rubber stopper for two or three hours with safety; but a longer exposure should be avoided, as moisture is gradually absorbed frum the air permenting the stopper. 
platinum vessel is altered, and consequently this vessel may not be in such intimate contact with the disk at one time as at another, and the amount of heat absorbed by the latter will therefore not always be the same. The error thus introduced, however, cannot be very large, since the disk was never found to be equivalent to more than one hundredth of the contents of the calorimeter.

It was thought possible that an error might also arise in the following manner. Before the introduction of any salt into the calorimeter, all the parts of the apparatus are at the same temperature; but as soon as a reaction takes place, involving, say, the evolution of heat, the radiation from the calorimeter will raise the temperature of the vessel which is interposed between it and the water-jacket (owing to its great bulk the water-jacket itself remains practically unaltered in temperature). During the rest of the time allowed for the experiment this vessel will therefore be at a higher temperature than it was initially; and consequently the rate of cooling during the last intervals will be less than during the first one, the correction to be applied for cooling will be under-estimated, the intermediate vessel having in reality acted as so much calorimetric water. In order to ascertain whether the error from this source attained appreciable dimensions, four experiments with potassium sulphate were performed; in two of these the intermediate vessel was present as usual, while in the other two it was dispensed with. The results obtained were as follows:-

A. With the intermediate vessel :-

$$
\left.\begin{array}{l}
\text { 1. ...... }-7215 \text { calories } \\
\text { 2. ..... }-7181, "
\end{array}\right\} \text { Mean }-7198 \text { cal. }
$$

B. Without the intermediate vessel :-

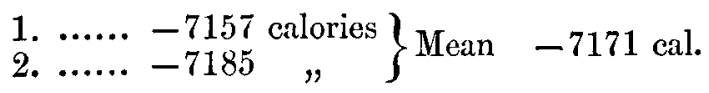

Showing that the use of the intermediate vessel had no appreciable effect on the results obtained *. Where, however, lirge heat-disturbances take place in the calorimeter, it is safer to dispense with it entirely; and indeed the employment of it is in no case attended by any advantage.

The time required to complete the dissolution of the salt varied, of course, in different cases, but rarely exceeded 5 minutes. The loss (or gain) of heat by radiation \&c. during this interval was determined separately in each experiment.

* If otherwise, the results of $A$ would be smaller than those of $B$. 
It was found that with the apparatus employed, and with a difference between the temperature of the calorimetric liquid and that of the surrounding air not exceeding $2^{\circ} .5 \mathrm{C}$., the rate of cooling was practically constant throughout the $20-30$ minutes (and even for a considerably longer period) during which the experiment lasted; whereas for accurate measurements it was never safe to trust to the results of one experiment to determine the rate of cooling in another experiment, even though the two were performed within 10 minutes of each other, and apparently under exactly similar conditions. To determine the rate of cooling, therefore, all the operations (pounding, stirring, \&c.), performed during the first $\mathbf{5}$ minutes to effect the dissolution of the salt, were imitated as nearly as possible during a second, third, and sometimes even fourth and fifth similar intervals, the temperature being read at the end of each interval. The actual rate of cooling (or heating) rarely exceeded $0^{\circ} 04 \quad C$. for 5 minutes, and was generally much less.

In these experiments the proportion of salt to water taken was $1: 400$ molecules*, the actual volume of the latter being about $600 \mathrm{enb}$. centim. The chief salts operated on were the sulphates of sodium, potassium, lithium, copper, magnesium, potassium-magnesium, and potassium-copper, both hydrated and anhydrous.

The thermometers were all read by estimation by means of a lens to the twentieth part of the marked divisions, $i . e$. to 0.05 millim. $\dagger$; and one such estimation-figure, being equivalent to $\cdot 002^{\circ} \mathrm{C}$., makes a difference of about 16 cal. in the molecular heat of dissolution. I should not put the estimation-figure at less than one tenth of the marked divisions were it not that these experiments afford ample proof that some reliance can be placed on smaller amounts than this.

The heat-disturbance in the calorimeter varied between nil and $2^{\circ} .3 \mathrm{C}$., the average being rather less than $1^{\circ} \mathrm{C}$.

Out of some 600 determinations of this nature, I may

* With double salts $1: 800$; but, for the sake of comparison, these are here reduced so as to be comparable with the other experiments.

+ The divisions in some of the instruments measured rather more than 1 millim., namely 1.2 millim.; but, prorided sufficient attention be paid to a certain proportion between the breadth of the mercurial column and the distance between the divisions, there is no appreciable differerice between estimating the fractional portions of these two quantities. Besides practice, and uniformity in the lines themselves, nothing conduces so much to accuracy in estimation as a just proportion between the breadth of the mercurial column and the distance between the divisions. I find the best proportion to be $1: 10 ; 1: 15$ is too great, and $1: 5$ too small. Different ratios, however, might suit different observers. 
discard the first hundred as consisting chietly of preliminary and imperfectly conducted experiments ; the next century of determinations was also found to give an experimental errov decidedly higher than the rest; these also I have therefore omitted, and will confine myself to the remaining 400 experiments. Out of these, however, it will only be the ones which were performed in duplicate which will be arailable for the present purposes.

Now the average difference between two such duplicate experiments, performed on the same day and with one thermometer only in the calorimeter, was found to be $26^{\circ} 6$ calories (deduced from 76 separate experiments), giving a probable error in each experiment of \pm 13.3 cal.; this corresponds to an error in the thermometric column of considerably less than $\pm^{\circ} 05$ millim.; and as the results involve not only' four or five readings of the thermometer, but also the correct reproduction of every action performed in dissolving the salt during the intervals allowed for the determination, of the rate of cooling, the correct determination of the salt temperature, and many other points where serious experimental error may occur, I consider that I am fully justified in concluding that the thermometers can be read to .05 millim., and that even a certain amount of reliance can be placed on such estimation figures.

Where the duplicate experiments were not performed on the same day, the error was found to be greater than in the previous case ; on an average, deduced from such experiments, it amounted to \pm 19.6 cal. This number is, however, somewhat bigger than it should be for strict comparison with the previous one; for the experiments which yielded it belonged chiefly to the earlier ones amongst the 400 under consideration, and an increase of accuracy appeared to be made with increased practice throughout the whole series.

In what follows I deal with those duplicate experiments which were performed consecutively only. We thus have so far:-

I. Difference of two experiments where one thermometer was employed, $26 \cdot 6$ cal.

Now in a large number of cases two thermometers were used in the calorimeter at the same time; from these was deduced,

II. Difference of two experiments where two thermometers were employed, taking the results obtained with the same instrument in the two experiments, $41.9 \mathrm{cal}$. (from 72 experiments).

Subtracting I. from II., we get 
III. 15.3 cal. as the increase of difference in two experiments due to the increased difficulty of manipulation when two thermometers are used instead of one.

IV. Difference in two experiments when two thermometers are used, comparing the results obtained with the one instrument in the one experiment with those of the other instrument in the other experiment, 53.02 cal. (from 76 experiments).

V. Difference in the results obtained from the two different thermometers in the same experiment, 33.95 cal. (from 142 experiments),

Now, subtracting II. from IV., we get

VI. That portion of the difference in the results given by two thermomeiers in any single experiment which is due to the instruments not being absolutely concordant, $11.12 \mathrm{cal}$.

(This amount will represent the sum of the errors in the calibration and standardization of the instruments. As it is dependent on two readings of each instrument, the amount attributable to each reading will be $3.7 \mathrm{cal}$, corresponding to $\cdot 014$ millim., or $0 \cdot 00056^{\circ} \mathrm{C}$.)

Subtracting this amount from V. gives

VII. Difference due to the error inherent in reading the two thermometers, $22.83 \mathrm{cal}$, and therefore a difference due to error in reading each thermometer of $11.42 \mathrm{cal}$.

Subtracting these two quantities from I. and II. respectively, and halving the results, we obtain :-

A. In a single experiment where one thermometer is used:-

(1) Error of reading ............. \pm 5.71 cal.*

(2) Error due to manipulation ... \pm 7.59 ,

$$
\text { Total ............. } \overline{ \pm 13 \cdot 3} \text {, }
$$

B. In a single experiment where two thermometers are used:-

(1) Error of reading .............. \pm 11.42 cal.

(2) Error due to manipulation ... \pm 9.53 ,

$$
\text { Total .............. } \overline{ \pm 20.95}
$$

Theso duplicate experiments being performed at exactly the same temperature, no difference will appear between them due to errors of calibration or standardization ; but in regarding the absolute value of the numbers deduced from them, this source of error must also be taken into account. The amount of this error has already been shown to be 11.12 cal. when the results of two instruments are compared (VI.); the total

* Corresponding to .021 millim. of the mercurial column. 
error, therefore, in experiments where one thermometer is used will have to be increased by half this amount, thus becoming +18.86 cal. This number, then, represents the probable divergence from the truth of any one experiment; and, from the manner in which the various thermometers were standardized (by comparison with each other), this error will probably be as great when two experiments performed at different temperatures are compared, whether they were performed with the same or with different instruments.

Perhaps, for the purpose of determining this probable error, the calculations ought to be based on the difference between duplicate experiments performed not on the same day, in which case the error would be somewhat larger. I have not done so, however, chiefly owing to uncertainty in the number given for this quantity (see above). Many causes also which tend to increase the difference when the experiments are not performed consecutively might not apply in general. Amongst these I may mention the very hygroscopic nature of many of the salts taken, where a difference in the state of moisture of the atmosphere would affect the extent to which traces of water were absorbed during their exposure while being transferred to the weighing-tubes: these substances, again, were often kept in sealed tubes containing sufficient for two or three determinations only, and the contents of the various tubes may not have been dried to precisely the same degree.

XLV. On Delicate Calorimetric Thermometers. By SPENCER Umpreville Piokerlang, M.A., Professor of Chemistry at Bedford College*.

T $\mathrm{N}$ the foregoing communication it has been shown that the average error of a determination of the heat of dissolution of a salt in $400 \mathrm{H}_{2} \mathrm{O}$ amounts to \pm 18.86 cal.; but this is only an average, and it must not be overlooked that the error in some cases may exceed this very considerably. It is not uncommon to find a difference of 40 or $50 \mathrm{cal}$. between two duplicate experiments (although the average error in such cases is only \pm 13.3 cal.), and a proportionately larger error must therefore be expected occasionally in the comparison of two experiments at different temperatures.

The determinations from which these conclusions were drawn consisted of a series of experriments with various salts at temperatures ranging from $1^{\circ}$ to $26^{\circ} \mathrm{C}$, where it was imperative that the greatest possible accuracy should be attained, and also that the experiments at the different temperatures should all be strictly comparable with each other. 'The ther-

* Communicated by the Physical Society : read February 13, 1886. 